\title{
Exploration of Private Information Market Mechanism
}

\author{
Wenjun Zhang \\ North China Electric Power University. Baoding Hebei, China \\ 305835338@qq.com
}

\begin{abstract}
In the paper, we present supply model, demand model and the pricing structure under the condition of equilibrium market. Firstly, we define the supply model and demand model being related to the risk extent through analyzing the quantity of the same private information for the same demander is only one or zero. Meanwhile, we also make comparisons both of individual and market in the aspect of supply and demand. Secondly, we make an analysis of supply model and demand model in the market condition. In order to analyze the change of the market price, we put it into shortrun and long-run condition separately. Then we find suppliers will have extra profit in the short time, and only in the long run can both of suppliers and demanders reach the maximal benefits. Further, we also consider the influence of the perception in different age group and the intensity of network effects to the pricing structure.
\end{abstract}

Keywords: Private information, Market mechanism, Equilibrium market.

\section{Introduction}

As I said in my previous paper Exploration of Private information Pricing Structure, some people seem willing to share PI about their personal interactions, relationships, and so on. Nowadays, PI is more and more likely to be a kind of special goods that can be priced and sold, just like the phone answerer we just mentioned above. PI gradually become a special commodity.

We have presented the private information Pricing Structure, now we want to know more about what will happen when PI is sold as a commodity in the market, and we define Supply Function and Demand Function to describe this special commodity in the paper.

\section{Exploration of Market Mechanism}

\subsection{Supply Funciton}

Supply Funciton is the point trajectory of diverse risk extent of information for sale and its corresponding price $\left(P_{s}\right)$

First of all, we may distinguish the essence of private information in each trade: it is just some kind of private information. Everyone has large quantity and variety of private information, such as name, telephone, personal reputation, assets and so on.

Each kind of information means different goods for Demanders, so they will have different preference. Meanwhile, Each kind of information only one for the same Demander, it means when the Demanders purchase the information, they have no way to get more. It can be expressed by the equation below:

$$
Q_{d}=\left\{\begin{array}{l}
0, \text { purchased } \\
1, \text { no } \text { purchase }
\end{array}\right.
$$

So, the price of each kind of information is fixed for the same Demander, which means it may not be explained simply by the Supply function in microeconomics [1].

Therefore, we define the Supply Funciton to describe he relationship between the risk of information for sale and its corresponding price $\left(P_{s}\right)$.

As I mentioned in my previous paper, we define the Risk Function is

$$
R=\boldsymbol{\alpha} \cdot \mathbf{s}^{\mathbf{T}}
$$


Where

$$
\boldsymbol{\alpha}=\left[\alpha_{1}, \alpha_{2}, \alpha_{3}\right], \mathbf{s}=\left[s_{1}, s_{2}, s_{3}\right]
$$

So, we can define the supplier price is equal to the risk cost of trading private information obviously. So, we can know Supply Function of different person:

$$
P_{S}=C=\sum_{i=1}^{n} R_{i} \cdot m=\sum_{i=1}^{n} R_{i} \cdot \prod_{i=1}^{n} R_{i}^{\beta_{i}(n)}
$$

Distinguishingly, when the supplier only sells one piece of information in a trade, it may be renewed:

$$
P_{S}=C=R
$$

\subsection{Individual Supply and Market Supply}

The shape of the Supply Funciton depends on the character of person, so the individual Supply Funciton is equal to the market Supply Funciton, which means the amount of people who sell information are the number of Supply Funciton in the market.

\subsection{Demand Model}

Demand Funciton is the point trajectory of diverse risk extent of purchased information and each of corresponding expectancy price $\left(P_{u}\right)$.

As mentioned above, the quantity of goods in private information market is one or zero, which means it difficult to explain the Demand relation in PI market by Demand function in microeconomics, thus, by reference to process of providing the Supply Funciton mentioned above, we come up with a Demand Funciton suitable for PI.

First, we may find the expectancy utility (or profit) related to the risk extent of purchased information:

$$
U=U(V)
$$

Where $V$ is the added value.

Then, according to the assumption of "Second sale is prohibited", we may infer that the Demander price, which we name it as utility price here, is equal to $U$, so we get:

$$
P_{u}=U=U(V)
$$

And we get the figure in $P_{u}-V$ coordinate system:

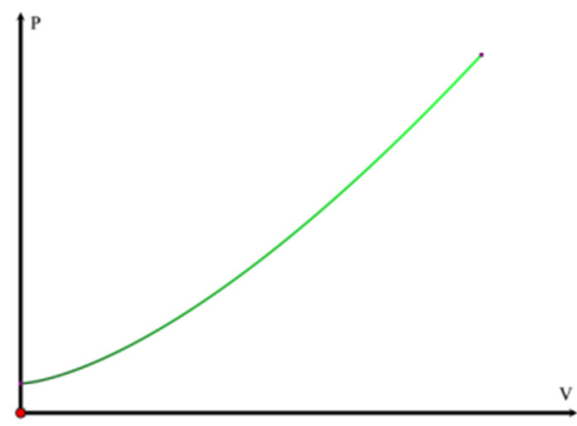

Fig 1. Demand Price-Added Value

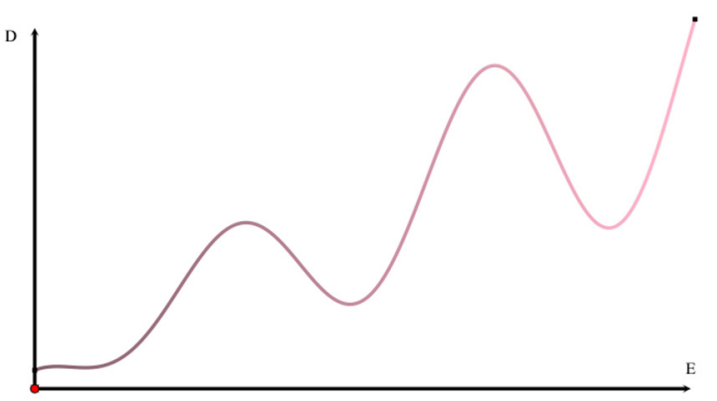

Fig 2. Demand-Task Extent 
However, we should pay attention to the fact that the utility (or profit) is not positive correlation with the risk extent. There is a simple example to explain this fact: The information that having AIDS is very vital for you, but to the Demander, it maybe just means they get one more profit. It is obviously that the risk extent of the supplier is not equal to the utility (or profit) of the Demander. So, we can get another figure in (which $\mathrm{R}$ means risk-extent) coordinate system like figure 2.

Because of the limited data, we can't find concrete equation between expectancy price and risk extent, so we only give the rough relationship below:

$$
P_{u}=P_{u}(R, \varepsilon)
$$

Where $R$ is the risk Function, $\varepsilon$ is the effect of random event.

\subsection{Individual Demand and Market Demand}

Equally, because the added value different of different information is relate to the Demanders' domain, which means that the kinds of the Market Demand are dependent on the kinds of Demander.

\subsection{Pricing Structure under Equilibrium Market Condition}

After the deducing process and analysis, we have had Supply Funciton and Demand Funciton about private information. Next we take both of their figure into the same coordinate system, and exploring the pricing structure under short-run and long-run equilibrium market condition.

\subsection{Pricing Structure under Short-run Equilibrium Market Condition}

In the short-run market, we consider the price of both supplier and Demander is fixe. Therefore, with the difference between the risk extent of supplier and the expectancy utility of Demander, it well appears the situation of the difference in market price like figure 3 below.

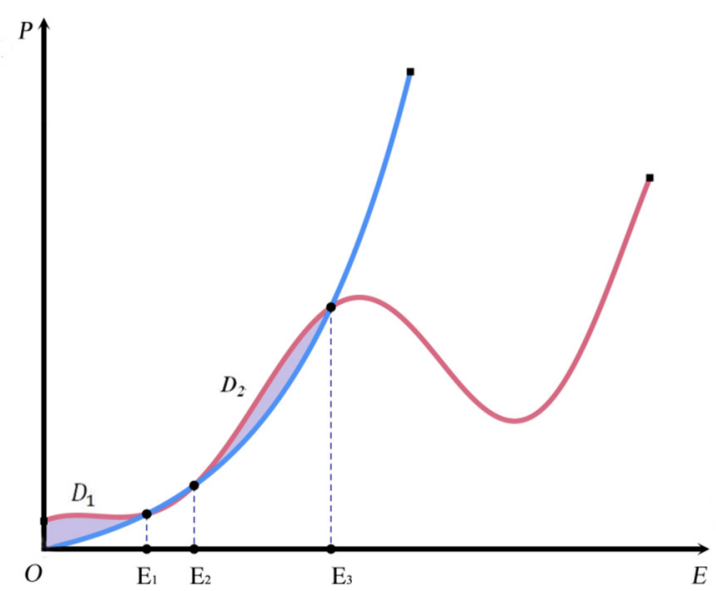

Fig 3. Supply-Demand in Short-run

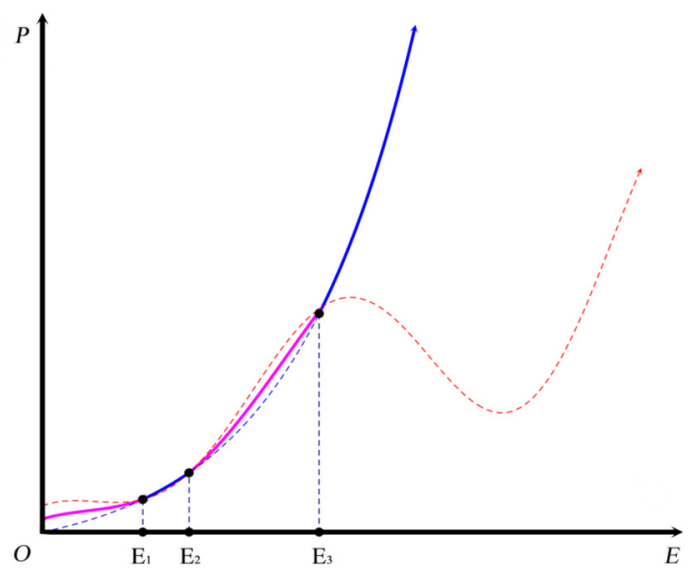

Fig 4. Supply-Demand in Long-run

When in $O E_{1}$ and $E_{2} E_{3}$ segment, the Demanders' price is high. But in $E_{1} E_{2}$ and after $E_{3}$ segment, the suppliers' price is higher than Demanders'. Further, we can find the price of supplier is above Demanders' price when the risk extent tend to positive infinity, the reason is the difference between the risk extent of supplier and the expectancy utility of Demander. There doesn't exist the situation of expectancy utility tending to positive infinity with the increasing of risk extent.

With the market experience, supplier must sell the private information when the $P_{u} \geq P_{s}$ so we can derive the pricing structure under short-run equilibrium market condition: 


$$
\text { price }_{\text {short-run }}=\left\{\begin{array}{l}
P_{s}=\sum_{i=1}^{n} R_{i} \cdot \prod_{i=1}^{n} R_{i}^{\beta_{i}(n)}, E \in\left[\left(E_{1}, E_{2}\right) \cup\left(E_{3},+\infty\right)\right] \\
P_{u}-P_{s}=P_{u}(R, \varepsilon)-\sum_{i=1}^{n} R_{i} \cdot \prod_{i=1}^{n} R_{i}^{\beta_{i}(n)}, E \in\left(D_{1} \cup D_{2}\right)
\end{array}\right.
$$

\subsection{Pricing Structure under Long-run Equilibrium Market Condition}

From figure 3, we can find both supplier and Demander are not in the situation of maximize benefits, which leads to the price changeable in the long-run market. So, the price is different between short-run market and long-run market.

When in long-run market, we assume the price is variable and the time is long enough to make both supplier and Demander into new equilibrium like figure 4.

It is normal for suppliers to raise price and for Demander to depress price. With the two charges at same time, the market price will be a new equilibrium in the end $\left(P_{e}\right)$. But because of the limited data, we can only draw the Supply-Demand equilibrium curve above.

$$
\text { price }_{\text {long-run }}=\left\{\begin{array}{l}
P_{s}=\sum_{i=1}^{n} R_{i} \cdot \prod_{i=1}^{n} R_{i}^{\beta_{i}(n)}, E \in\left[\left(E_{1}, E_{2}\right) \cup\left(E_{3},+\infty\right)\right] \\
P_{e}, E \in\left[\left(0, E_{1}\right) \cup\left(E_{2}, E_{3}\right)\right]
\end{array}\right.
$$

Where $P_{e}$ is constrained by $P_{s}<P_{e}<P_{d}$.

\section{Conclusion}

In the paper, we define Supply Function and Demand Function, and we present the relationship between individual supply/demand and market supply/demand.

we discuss the application of demand function and supply function in equilibrium market. Meanwhile, we also consider the differences between the two functions under different equilibrium periods such as short-run equilibrium and long-run equilibrium, and present their equilibrium function.

\section{References}

[1]. Li Wei. Microeconomics[Z].2013.

[2]. Wu Ying, Li Rui. Survey of cognition status quo of physical examination people at different ages on privacy right [J]. The Second Militany Medical University, Shanghai 200003, China.2017. 